\title{
Supplementary Materials to: Climatic information archived in ice cores: impact of intermittency and diffusion on the recorded isotopic signal in Antarctica
}

M. Casado, T. Münch, T. Laepple

1. Climatic parameters
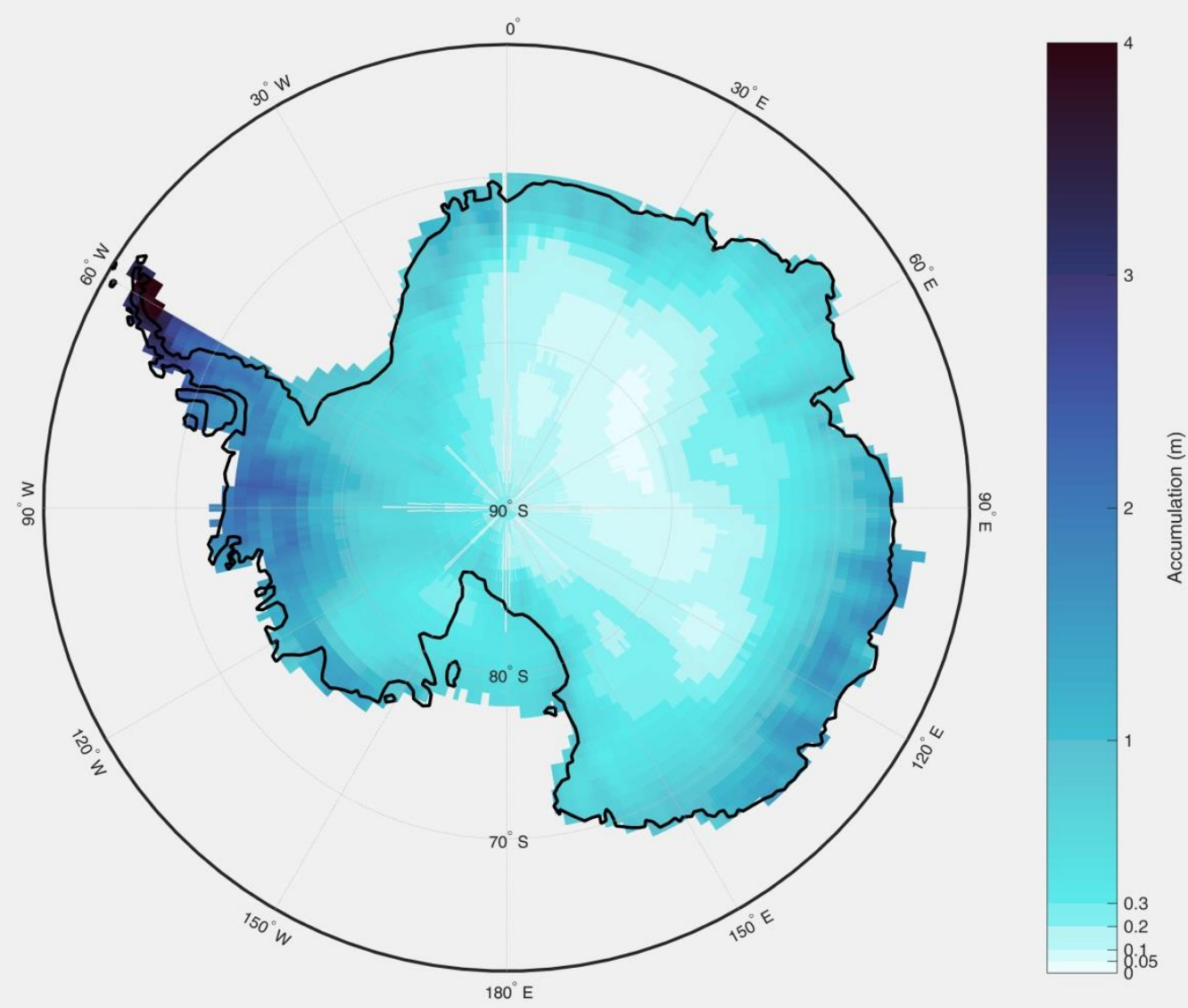

Figure S1: Maps of the accumulation rate (m. $\left.\mathrm{a}^{-1}\right)$ produced by correcting ERAinterim precipitation amounts with satellite data and ice core estimates

2. Impact of the accumulation correction

We run the model at EDML without including the accumulation amount correction. The noise level added by precipitation intermittency, without the accumulation amount correction is $0.65 \%{ }^{2} \mathrm{~m}$, slightly above the value obtained when the accumulation amount correction is implemented $\left(0.59 \%{ }^{2} \mathrm{~m}\right)$.

This results in small increase of the time scales at which a meaningful signal can be retrieved (see Fig. S2). 


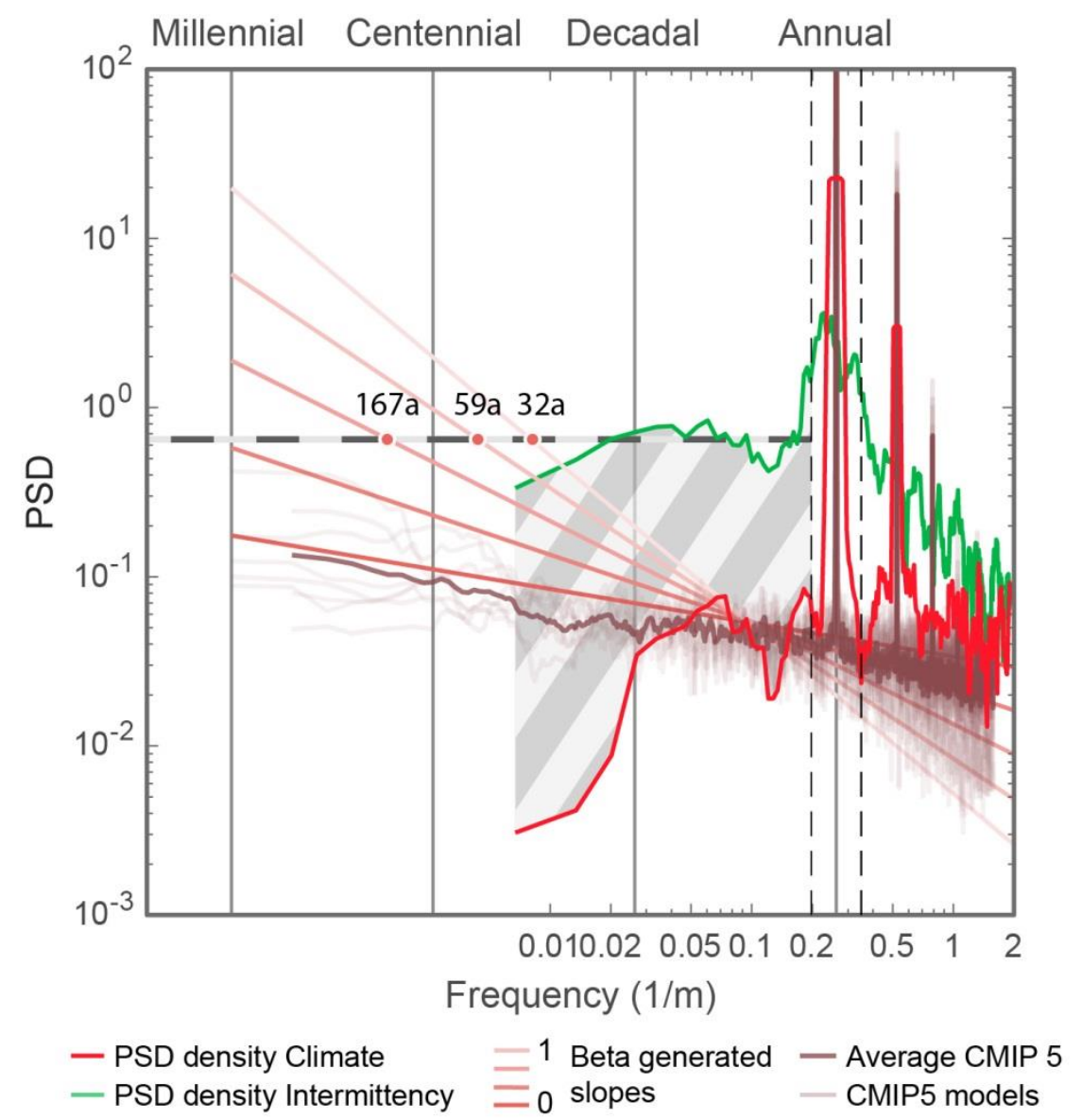

Figure S2: Same as Figure 4 without the correction of the accumulation.

When including diffusion, we obtain values for $\tau_{a}$ of 77 and 18a for values of $\beta$ of 0.6 and 0.8 , respectively. While these values are larger than the ones obtained when the accumulation is corrected to be matching observations, they remain of the same order of magnitude, even though the accumulation rate is 17 times stronger than the observations. This extreme case shows that the model performances can satisfy sensitivity tests.

\section{Impact of the perfect dating assumption on the power spectral estimates}

The perfect dating assumption consists of tagging each layer of snow with its date. In this manuscript, we used this property directly in the time domain to transfer from depth to time. In the spectral domain, because the phase are erased, the transfer from a depth scale to a time scale can be done by converting directly using the accumulation rate. This conversion yet still hides the perfect dating assumption when used to compare the climatic signal to virtual core.

We evaluate the impact of the perfect dating assumption on the power spectral estimates of the amount of noise added by precipitation intermittency by reproducing the calculation with PSD of time series (transferring from depth to time series and interpolating to a daily resolution), compared to depth series before. 


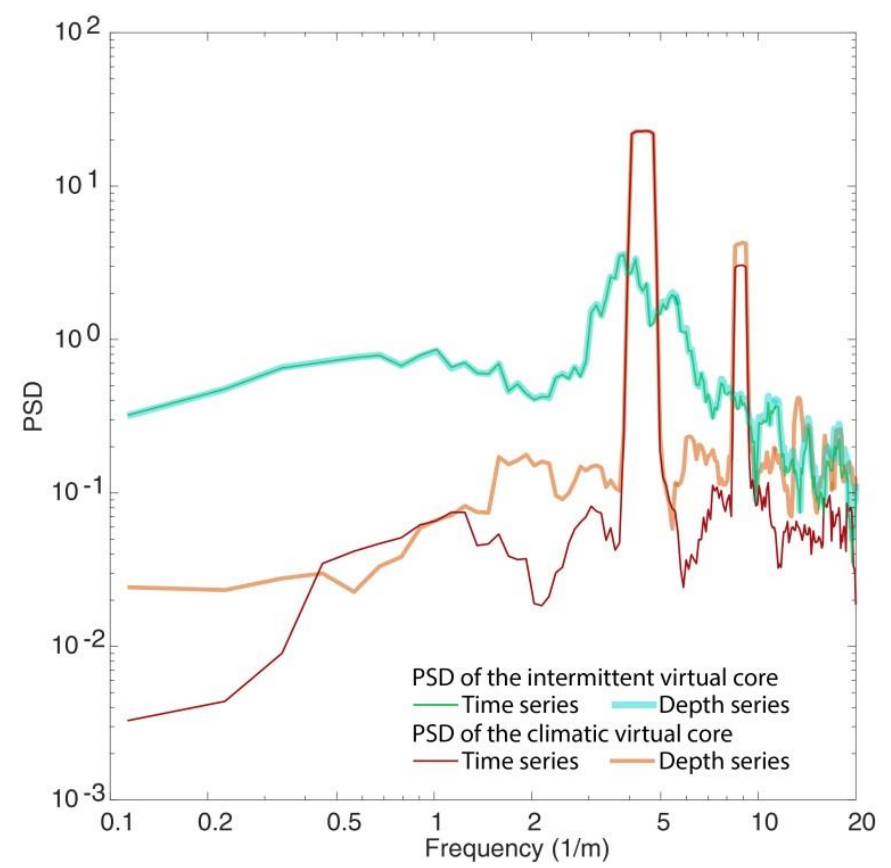

Figure S3: Power spectral density of the intermittent virtual core and the climatic virtual core calculated from time series (dark fine lines) or depth series (light large lines).

Some differences are mainly visible in the PSD of the climatic virtual core. Overall, the amount of noise added by precipitation intermittency is slightly larger when the calculation is done from time series $\left(0.63 \% \mathrm{o}^{2} \mathrm{~m}\right)$ compared to the calculation done from depth series that we used in this manuscript $\left(0.59 \%{ }^{2} \mathrm{~m}\right)$. These results account for changes of the calculation of $\tau_{a}$ of less than $20 \%$ 